\title{
A collaborative clinic between contraception and sexual health services and an adult congenital heart disease clinic
}

\author{
Paula Rogers, Diana Mansour, Alison Mattinson, John J O’Sullivan
}

\begin{abstract}
Background The success of cardiac surgery has created a new group of patients: those with 'adult congenital heart disease' (CHD) who may need specialist advice about contraception and pregnancy. The study objective was to investigate whether women with CHD were receiving appropriate advice on contraception.
\end{abstract}

Methods The study setting comprised a combined adult $\mathrm{CHD}$ and contraception and sexual health clinic operating alongside each other, once a month, at Freeman Hospital, Newcastle-upon-Tyne, UK. Data were collected on 46 consecutive female patients attending the clinic between April 2002 and October 2003.

Results Sixteen of the 46 (35\%) women had never discussed contraception with a health professional. Nine of these women were sexually active and were using condoms. Seven of these women chose to start hormonal contraception following consultation. Ten of the 30 (33\%) women who had previously discussed contraception with either their general practitioner or family planning clinic had received inappropriate advice. Of these 30 women, 24 needed contraception: $12(50 \%)$ continued with their current method, 10 (42\%) started hormonal contraception having previously used either condoms or no contraception and two (8\%) changed their current hormonal method to a more effective long-term method (progestogen-only pill to progestogen implant). There had been eight unplanned pregnancies in seven patients. There was poor knowledge among the women about longacting hormonal methods, particularly progestogen injectables and implants.

Conclusions Our experience has highlighted the substandard provision of sexual health services for adults with CHD. Many of these women receive either no advice or inappropriate advice about contraception. Suitable effective reversible methods are often denied by health professionals who are concerned about the safety of hormonal contraceptives in women with 'heart problems'. Preconception advice and birth control information should be given to all female patients with $\mathrm{CHD}$, as correct information will avoid the potential risks of an unplanned pregnancy. A monthly regional combined clinic staffed by a cardiologist and family planning doctor provides the ideal opportunity for education of patients and health professionals alike. Good practice is then disseminated across specialities and into the community, encouraging multidisciplinary guidelines and pathways of referral to be developed.

Keywords cardiology, congenital heart disease, contraception, long-acting reversible contraception, sexual health services

J Fam Plann Reprod Health Care 2007; 33(1): 17-21

(Accepted 26 September 2006)

\section{Introduction}

Contraception and sexual health services are well developed throughout the UK. Even so, contraceptive choices are often restricted when women give a complicated medical history. This is particularly true for patients with congenital heart disease (CHD).

Over the last 25 years, a new group of patients has been created owing to the success of paediatric cardiac surgery. Many more 'congenital heart patients' become young adults under the care of paediatric cardiologists. A paediatric cardiology consultant is used to dealing with children and may be unaware of their patients' transition into sexually mature individuals, often at young ages. ${ }^{1}$ Contraceptive advice and effective provision for women

\footnotetext{
Department of Contraception and Sexual Health, Graingerville Clinic, Newcastle General Hospital, Newcastle-upon-Tyne, UK

Paula Rogers, MBBS, Career Grade Registrar

Diana Mansour, FRCOG, FFFP, Consultant in Community

Gynaecology and Reproductive Health Care

Alison Mattinson, MBBS, FFFP, Associate Specialis
}

Department of Congenital Heart Disease, Freeman Hospital, Newcastle-upon-Tyne, UK

John J O'Sullivan, FRCPI, Consultant

Correspondence to: Dr Paula Rogers, Graingerville Clinic,

Newcastle General Hospital, Westgate Road,

Newcastle-upon-Tyne NE4 6BE, UK.

E-mail: paularogers@doctors.org.uk

\section{Key message points}

- A growing number of adults in the UK with congenital heart disease (CHD) require contraception and sexual health advice.

- One-third of the women in the present study had been given poor or conflicting contraceptive advice by general practitioners or family planning clinics.

- Women with CHD have little knowledge of effective, reversible long-acting contraceptive methods.

- Health professionals are often over-cautious and deny women with CHD effective and safe contraceptive methods.

- A regional service working with cardiologists improves contraception and sexual health advice and increases the use of long-acting contraceptive methods in these women.

with CHD is essential to reduce life-threatening complications that may arise from an unwanted pregnancy.

In the community family planning clinic setting without access to general practitioner (GP) records or indeed cardiology records, it is very difficult to make an informed decision about which contraceptive method is suitable for a particular patient. The increased risk of arterial or venous thromboembolism with hormonal birth control methods cannot be predicted from the 'cardiac diagnostic label' alone. Often patients have to be sent away while further information is sought. This can be time consuming and can take weeks, with letters going back and forth between the community clinic, general practice and cardiologists. In the 
case of an adolescent, an opportunity may be missed and the patient left exposed to the risk of pregnancy.

However, a busy cardiology outpatient clinic is not the best place to discuss sexual health issues. It is often difficult for paediatric cardiologists to provide adequate and up-to-date contraceptive advice, as they have neither training nor adequate experience in this field. ${ }^{2}$ Many women with CHD receive incorrect contraceptive advice and are unaware of the family planning choices available to them. ${ }^{1,3}$ It is against this background that a contraception and sexual health clinic operating alongside an adult CHD clinic was proposed.

\section{Methods}

A combined adult CHD and contraception clinic is held every month at Freeman Hospital, Newcastle-upon-Tyne, UK. This specialist, adult, CHD centre deals mainly with patients at the moderate or severe end of the CHD spectrum since most patients with mild lesions are discharged from the paediatric cardiology clinic.

At the regional combined contraception clinic the cardiologists book in mostly female patients. Data are collected prospectively. The cardiologist sees each patient and assesses the risk a pregnancy would pose and the suitability of different contraceptive methods. The cardiologist has a detailed knowledge of the patient's past history and also carries out a clinical examination and echocardiogram at this clinic visit. Certain conditions are regarded as clear contraindications to certain therapy (e.g. estrogen-containing contraception in patients with Eisenmenger's syndrome). However, each patient is assessed individually and the recommendations are not based on a 'headline' diagnosis. The family planning doctor reviews the patient and discusses all suitable contraceptive options. If the method chosen is considered absolutely or relatively contraindicated, further

Patient arrives at combined congenital heart disease and contraception clinic

\section{Initial consultation with cardiologist}

- Cardiac history and examination

- Echocardiogram

- Patient asked if she would like to see a family planning doctor

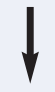

Discussion between cardiologist and family planning doctor

Main points discussed are:

- Cardiac diagnosis and medication

- Risk of arterial/venous thrombus and contraindications to estrogen-containing methods of contraception

- Risk of endocarditis and contraindications to intrauterine methods of contraception

- Risk of an unplanned pregnancy to patient's cardiac condition and risks to fetus

\section{Consultation between patient and family planning doctor}

- Sexual history

- Contraceptive methods discussed

- If required, contraception prescribed or arranged for a suitable date

- With patient's consent a letter is sent to her general practitioner outlining the consultation

Figure 1 Summary of management of patients attending the combined congenital heart disease and contraception clinic
Table 1 Age range of patients included in the survey

\begin{tabular}{lc}
\hline Age range (years) & $\boldsymbol{n}$ \\
\hline$<16$ & 1 \\
$16-19$ & 18 \\
$20-24$ & 10 \\
$25-29$ & 7 \\
$30-34$ & 3 \\
$35-39$ & 5 \\
$40-45$ & 2 \\
\hline
\end{tabular}

discussion takes place with the cardiologist. A contraceptive plan is formed and discussed with the patient based on the cardiac lesion and risk of unplanned pregnancy.

When appropriate, other sexual health issues are also discussed. In addition to providing all contraceptive methods, the clinic provides cervical screening, pregnancy testing and referral for termination of pregnancy. If a patient decides to have an intrauterine device (IUD) or a levonorgestrel-releasing intrauterine system (IUS), initial assessment and chlamydia screening are performed at the combined clinic and an appointment for insertion at the central contraception and sexual health clinic is arranged. It is not always possible to administer the first progestogen injection or insert a contraceptive implant on the same day owing to previous risk of pregnancy during that menstrual cycle. A subsequent visit or appointment at the patient's local family planning clinic is organised.

With the patient's consent, a letter is sent to their GP covering all aspects of the consultation including the final method of contraception chosen.

A summary of patient management is shown in Figure 1.

\section{Results}

During the period April 2002 to October 2003, 17 clinics took place. Some 46 women aged from 15 to 42 years made a total of 47 visits. The mean age of patients seen was 24.2 years and the median was 21 years. The age breakdown for all patients is given in Table 1 .

\section{Current vs recommended contraception}

Sixteen of the 46 (35\%) women had never discussed contraception with a health professional. Five of these women had never been sexually active; two were not currently sexually active. Following consultation and discussion about suitable methods, these seven women decided to delay contraception until it was needed. The remaining nine women were all using condoms as their method of contraception. Following consultation, three women started on the progestogen-only pill (POP), three chose the progestogen implant, one chose the combined oral contraceptive pill and two decided to continue with condoms.

Thirty women (65\%) had previously discussed contraception with a health professional. Ten (33\%) of these women who had previously discussed contraception with either their GP or family planning clinic had received inappropriate advice:

- Four women had been unnecessarily denied any form of hormonal contraception, despite progestogen-only methods being safe to use. Two of these women decided to start a POP and one woman chose to have a progestogen implant inserted. The fourth woman was not currently in a relationship. She planned to consider a progestogen injection or implant when needed.

- Three women had been told that the combined pill was absolutely contraindicated with their medical condition. 
Following consultation and echocardiogram assessment with the cardiologist, the combined pill was an option available to these women. One woman had undergone surgical correction of tetralogy of Fallot; she was currently on a POP and happy to continue with this. One woman had mild aortic stenosis and was using condoms. After consultation she chose to start the combined pill. The third woman had mild mitral regurgitation and mild mitral stenosis. She had had an unplanned pregnancy at the age of 17 years while taking a POP. She was currently using condoms and following consultation decided to start the combined pill.

- Two women had been inappropriately prescribed the combined pill in the past. One had moderate mitral regurgitation, moderate aortic regurgitation and aortic stenosis and one had moderate aortic stenosis. Following echocardiogram assessment, the combined pill was contraindicated due to an increased risk of thrombus formation. Both women had recently stopped taking the combined pill. One had stopped due to her relationship ending. She decided to use the POP with her next relationship. The other woman had stopped as her partner had undergone a vasectomy.

- One woman had been told by her GP to stop the POP despite progestogen-only methods being safe to use. The combined clinic family planning doctor prescribed the POP once more.

Of the remaining 21 visits made to the combined clinic by women who had previously sought advice from a health professional, nine women continued with their current method of contraception. Four women started the POP having previously used no contraception or condoms, one woman started on the combined pill having previously used no contraception, two women changed from the POP to the progestogen implant, two women who were not currently sexually active received advice on future contraception, one woman was trying to conceive and was given preconception advice, one woman was pregnant and one woman had psychosexual problems.

\section{Women choosing more effective contraception following consultation}

Of the 16 women discussing contraception for the first time, nine were sexually active. They were all using condoms as their main contraceptive method. Seven of these nine (78\%) women chose to start hormonal contraception and the other two continued with condoms. Of the remaining 30 women seen at the combined clinic, 24 needed contraception. Twelve $(50 \%)$ women continued with their current method of contraception. Ten (42\%) women started hormonal contraception, having previously used either condoms or no contraception. Two (8\%) women changed their current hormonal method to a more effective long-term method (i.e. POP to progestogen implant).

\section{Unplanned pregnancies}

There had been eight unplanned pregnancies in seven $(15 \%)$ of the women.

- Four women were using condoms at the time of conception. Three were still using condoms when they attended the clinic. Following consultation, two chose to have the progestogen implant and one chose to start the combined pill. The fourth woman had developed severe psychosexual problems following a termination of pregnancy 11 years previously. She was in a 10-year relationship and had decided to abstain from sexual intercourse due to her fear of pregnancy. This fear had never been addressed. She was offered referral to a psychosexual counsellor.

- One woman had two unplanned pregnancies while taking the combined pill. Her degree of compliance was uncertain. She was using condoms when she attended the clinic and continued with this method as she was planning a pregnancy in the near future.

- Two women were not using any contraceptive method at the time of conception. One woman was still not using any contraception. Estrogen and intrauterine methods were contraindicated and following consultation she decided to consider progestogen-only methods. The other woman, a 16-year-old, had had a termination of pregnancy 1 month prior to attending the combined clinic. She had not had sexual intercourse since the procedure. Following consultation, she chose to have a progestogen implant.

\section{Patients' knowledge of available contraceptive methods}

Twelve (26\%) women were aware of all the methods available. Most of the other women had poor knowledge of progestogen-only methods, especially injectables and implants.

\section{Discussion}

There is little published information about safe use of contraceptive methods in adults with CHD. This has led to extreme caution being exercised by GPs and family planning doctors as illustrated by the present audit. It is impossible to tell from the cardiac diagnostic label alone if hormonal contraception, in particular the combined pill, is contraindicated. One-third of women who had previously discussed contraception with their GP or family planning clinic had been given inappropriate advice. Of those 10 women, five $(50 \%)$ had been denied any form of contraception despite progestogen-only methods being safe to use. Of the seven women who had an unplanned pregnancy, six $(86 \%)$ continued to use a suboptimal method of contraception (condoms or no method) to prevent further pregnancy. The remaining woman was not having sexual intercourse due to psychosexual problems following a termination of pregnancy.

Another striking observation was the lack of knowledge amongst these women about progestogen-only methods, especially injectables and implants. These highly effective yet reversible contraceptive methods are very suitable for women with complex heart problems. The clinic was successful in educating attendees and providing effective contraception. Seventeen (37\%) of the women started hormonal contraception, having previously used either condoms or no method. Two women changed their current method to a more effective long-term method (POP to progestogen implant)

Little is known regarding the absolute risks for these patients. Many of the current recommendations are based on anecdote and common sense. 4 Informed, evidencebased advice remains difficult to give because of low absolute numbers of patients and the many types and degrees of cardiac anomalies and different therapies. ${ }^{5}$ The World Health Organization (WHO) has recently published eligibility criteria for contraceptive use and these will be referred to in subsequent paragraphs. ${ }^{6}$ Specific medical conditions or individual characteristics have to be measured against these criteria, thus helping the health professional to decide whether a contraceptive can be used without restriction or where the risks of use outweigh the benefits (Table 2). 
Rogers et al.

Table 2 World Health Organization criteria for contraceptive use

\begin{tabular}{llll}
\hline Category & With clinical judgement & With limited clinical judgement \\
\hline 1 & $\begin{array}{l}\text { A condition for which there is no restriction for the } \\
\text { use of the contraceptive method }\end{array}$ & Use method in any circumstance & Yes \\
2 & $\begin{array}{l}\text { A condition where the advantages of using the } \\
\text { method generally outweigh the theoretical or } \\
\text { proven risks }\end{array}$ & Generally use the method & Yes (Use the method) \\
3 & $\begin{array}{l}\text { A condition where the theoretical or proven risks } \\
\text { usually outweigh the advantages of using the } \\
\text { method }\end{array}$ & $\begin{array}{l}\text { Use of method not usually recommended } \\
\text { unless other more appropriate methods } \\
\text { are not available or not acceptable }\end{array}$ & No (Do not use the method) \\
$\begin{array}{l}\text { A condition which represents an unacceptable } \\
\text { health risk if the contraceptive method is used }\end{array}$ & Method not to be used & No \\
\hline
\end{tabular}

The combined pill is deemed inappropriate if it is considered that a rhythm abnormality, increased atrial size, sluggish flow in a venous conduit, poor ventricular function, pulmonary vascular disease or hyperviscosity would increase the risk of venous or arterial thrombus formation. ${ }^{1}$ To clearly define any of the above abnormalities of the heart an echocardiogram is essential, therefore caution needs to be applied when prescribing in the community. It is unclear whether the concurrent use of anticoagulants or antiplatelet agents reduces the risk of thromboembolism. ${ }^{4}$ The combined pill may also aggravate heart failure or upset anticoagulant control.5 The anticoagulant effect of warfarin is antagonised by estrogens and high-dose progestogens. Hence WHO has advised that the combined pill should not be used in these circumstances (WHO 4) ${ }^{6}$ although some experts suggest that this would fall to WHO 3 in anticoagulated women.

Intrauterine contraception use is restricted in many patients due to the fear of endocarditis. Streptococcus viridans and Neisseria gonorrhoeae endocarditis, although extremely rare, have occurred. 5 In particular, replacement of an IUD can be associated with significant bacteraemia from vaginal organisms 4-6 minutes after insertion of the new device. ${ }^{7}$

There are comprehensive publications from the Clinical Effectiveness Unit (CEU) of the Faculty of Family Planning and Reproductive Health Care regarding intrauterine contraceptives. ${ }^{8,9}$ The CEU, in common with WHO, have stated that use of an IUD or IUS in this situation is acceptable as the advantages generally outweigh the theoretical or proven risks (WHO 2). ${ }^{6}$ However, these recommendations are thought to be suboptimal for women in the UK by cardiologists treating women with anatomical abnormalities of the heart. The Newcastle Contraception and Sexual Health Service and the Department of Congenital Heart Disease have agreed on the following recommendations:

1. Women with a previous history of endocarditis or a prosthetic heart valve should not have an IUD or IUS inserted for long-term contraceptive use as the theoretical risks of further cardiac complications outweigh the benefits provided by these methods, especially as other safer and equally effective options are available (WHO 4).

2. Any woman with a cardiac lesion that has an associated risk of endocarditis should have antibiotic prophylaxis. This includes women with small ventricular septal defects, heart valve lesions or patent ductus who have no history of endocarditis (WHO 3).

The antibiotics to be used are those recommended in the current British National Formulary. ${ }^{10}$ In the absence of specific guidance, prophylaxis should be used for both IUD insertion and removal.
Liaison with the woman's cardiologist is also important before insertion of IUDs as they may recommend other options such as the progestogen implant or injectable when specific factors render a woman at high risk of complications. An IUD may also aggravate menorrhagia caused by anticoagulant use. However, an IUS may be ideal in this situation provided there is little risk of endocarditis.

Progestogen-only contraceptive preparations (i.e. oral, injectable or implants) are suitable for almost all patients with CHD, as there is no proven increased risk of venous or arterial thromboembolic events. ${ }^{11}$ WHO have therefore given these methods a classification of WHO 1. Some authors suggest that due to the possible fluid-retaining properties of progestogen injectables caution should be exercised in patients with impaired systemic or pulmonary ventricular function. ${ }^{3}$ However, we can find no evidence to support this statement.

There are concerns that excessive bleeding, bruising or haematoma formation may occur when inserting a contraceptive implant, an IUD/IUS or giving an injectable in women taking warfarin or heparin. There is little published evidence to support this concern ${ }^{12}$ but as there is a theoretical risk advice should be sought from a local haematologist, cardiologist or specialist in contraception. Sonmezer et al. ${ }^{13}$ looked at 12 women who were anticoagulated for prosthetic heart valves and who had suffered from ovarian bleeding from corpora lutea. The women were given depot medroxyprogesterone acetate and followed up for a mean period of 39.3 months. All 12 women suffered no further bleeding of this type as ovulation was suppressed. Anticoagulation was well controlled with no mention of excessive bleeding or problems at the injection site.

Overall, when considering contraceptive choice, the benefits of using an effective contraceptive in this group of women usually outweigh the risks associated with an unplanned pregnancy.

Female sterilisation is a highly effective contraceptive method but should be seen as a permanent choice. Even so, in many cases the benefits of sterilisation outweigh the risk of subsequent pregnancy. Unfortunately patients in whom pregnancy is contraindicated also have the highest general anaesthetic risk. A few gynaecology units offer laparoscopic clip sterilisation under local anaesthesia ${ }^{14}$ but often, for such high-risk women, their partners choose vasectomy although it must be remembered that the male partner may outlive his spouse and wish to start a family with a new partner in the future. An alternative method of female sterilisation by hysteroscopic occlusion of the proximal Fallopian tubes $\left(\text { Essure }^{\circledR}\right)^{15}$ may be of value for these patients as the procedure may be performed with sedation and local anaesthesia. 16 
Table 3 Issues to be addressed before or during pregnancy

- Severity of underlying disease

- Complications expected during pregnancy, puerperium, postpartum

- Risk of endocarditis

- Risk of maternal death

- Care for child if mother dies

- Risks to fetus: size, growth, abnormalities, death

- Fetal heart scan

- Genetic counselling

- Teratogenic drugs

- Place of delivery and obstetric care

- Method of delivery

- Need for hospital treatment during pregnancy

- State of health after delivery

- Number of pregnancies

- Sterilisation after pregnancy

- Cardiac care in puerperium

The aim of the combined clinic is to help women with CHD to postpone pregnancy until a time when it suits their medical and social needs. Discussion about sexual health issues should begin when they reach childbearing potential. Sexually transmitted infections, pelvic inflammatory disease, contraception and unplanned pregnancy may all cause complications, medically and psychosocially, for the woman with CHD and these areas should be included as part of a general medical assessment. This is difficult for the cardiologist as it is often outside their field of expertise. For the adolescent, offering a private setting away from over-involved parents will foster rapport and ensure confidentiality. ${ }^{17}$

Most patients with simple congenital heart lesions tolerate both pregnancy and contraception well. ${ }^{3}$ Patients with more complex lesions need informed advice on contraception including discussion of risks. Failure to give adequate advice can lead to unplanned pregnancy with potential life-threatening consequences. Congenital malformations now represent the most common cause of morbidity and mortality from heart disease in pregnancy. ${ }^{18,19}$ The combined clinic is working towards all pregnancies being planned following pre-pregnancy assessment. Table 3 suggests issues to be discussed during counselling before pregnancy as recommended by the British Cardiac Society. ${ }^{4}$ This table highlights the importance of a planned pregnancy in this high-risk population.

Our experience has demonstrated poor provision of sexual health advice for adults with CHD. There are many reasons why the current situation is unsatisfactory. Supplying a list of cardiac lesions where certain methods are unsuitable is prone to problems owing to individual variation. Denying or overemphasising the risk of contraceptive methods may result in significant harm for women at increased risk if they were to become pregnant. Contraception and sexual health clinics are often held in the evening, staffed by a variety of health professionals, thus making it difficult to access specialist cardiology expertise.

The sexual health needs of adult women with CHD should not be ignored. Improved service provision is essential and regional centres for CHD need to consider this aspect of their patients' care. In the Northeast of England a combined specialist clinic has established successful referral pathways. Repeat audits of contraceptive use and unplanned pregnancies, in conjunction with patient satisfaction surveys, will continue to be used to evaluate this service further.

Statements on funding and competing interests
Funding None identified.

Competing interests None identified.

References

1 Leonard H, O'Sullivan JJ, Hunter S. Family planning requirements in the adult congenital heart disease clinic. Heart 1996; 76: 60-62.

2 Somerville J. The Denolin Lecture: The woman with congenital heart disease. Eur Heart J 1998; 19: 1766-1775.

3 Swan L, Hillis WS, Cameron A. Family planning requirements of adults with congenital heart disease. Heart 1997; 78: 9-11.

4 British Cardiac Society. Grown-up congenital heart (GUCH) disease: current needs and provision of service for adolescents and adults with congenital heart disease in the UK. Heart 2002; 88(Suppl. 1): 1-14.

5 Seaworth BJ, Durack DT. Infective endocarditis in obstetric and gynecologic practice. Am J Obstet Gynecol 1986; 154: 180-188.

6 World Health Organization (WHO). Medical Eligibility Criteria for Contraceptive Use (3rd edn). Geneva, Switzerland: WHO, 2004.

7 Murray S, Hickey JB, Houang E. Significant bacteraemia associated with replacement of intrauterine contraceptive device. Am J Obstet Gynecol 1987; 156: 698-700.

8 Faculty of Family Planning and Reproductive Health Care Clinical Effectiveness Unit. FFPRHC Guidance (January 2004). The copper intrauterine device as long-term contraception. J Fam Plann Reprod Health Care 2004; 30: 29-42.

9 Faculty of Family Planning and Reproductive Health Care Clinical Effectiveness Unit. FFPRHC Guidance (April 2004). The levonorgestrel-releasing intrauterine system (LNG-IUS) in contraception and reproductive health. J Fam Plann Reprod Health Care 2004; 30: 99-108.

10 British National Formulary (No. 50). London, UK: British Medical Association and the Royal Pharmaceutical Society of Great Britain, September 2005; 268.

11 World Health Organization Collaborative Study of Cardiovascular Disease and Steroid Hormone Contraception. Cardiovascular disease and use of oral and injectable progestogen-only contraceptives and combined injectable contraceptives. Results of an international, multicenter, casecontrol study. Contraception 1998; 57: 315-324.

12 Frederiksen MC. Depot medroxyprogesterone acetate contraception in women with medical problems. J Reprod Med 1996; 41: 414-418.

13 Sonmezer M, Atabekoglu C, Cengiz B, Dokmeci F, Cengiz SD. Depot-medroxyprogesterone acetate in anticoagulated patients with previous hemorrhagic corpus luteum. Eur $J$ Contracept Reprod Health Care 2005; 10: 9-14.

14 MacKenzie IZ, Turner E, O'Sullivan GM, Guillebaud J. Two hundred out-patient laparoscopic clip sterilizations using local anaesthesia. Br J Obstet Gynaecol 1987; 94: 449-453.

15 Ubeda A, Labastida R, Dexeus S. Essure: a new device for hysteroscopic tubal sterilisation in an outpatient setting. Fertil Steril 2004; 82: 196-199.

16 Kerin JF, Carignan CS, Cher D. The safety and effectiveness of a new hysteroscopic method for permanent birth control: results of the first Essure pbc clinical study. Aust $N Z \mathrm{~J}$ Obstet Gynaecol 2001; 41: 364-370.

17 Stevens-Simon C. Providing effective reproductive healthcare and prescribing contraceptives for adolescents. Pediatr Rev 1998; 19: 409-417.

18 Siu S, Chitayat D, Webb G. Pregnancy in women with congenital heart defects: what are the risks? Heart 1999; 81: 225-226.

19 Confidential Enquiries into Maternal and Child Health (CEMACH). Why Mothers Die 2000-2002. Sixth Report on Confidential Enquiries into Maternal Deaths in the United Kingdom. London, UK: RCOG Press, 2004.

\section{Visit the Faculty Website at www.ffprhc.org.uk}

